\title{
Trois méthodes pratiques pour le dosage des espèces arséniées d'intérêt toxicologique : application à un cas d'intoxication mortelle par l'anhydride arsénieux
}

\section{Three practical methods for the determination of arsenic species of toxicological interest: application in a case of fatal intoxication by arsenic trioxide}

Larbi BENRAMDANE $^{*(1,2)}$, M. ACCOMINOTTI ${ }^{(1)}$, M. DRAOUI ${ }^{(2)}$

(1) Laboratoire de Biochimie, Toxicologie et Analyse des traces, Hôpital E. Herriot - LYON (2) Laboratoire de Chimie Analytique, Faculté de Médecine et de Pharmacie - RABAT - MAROC

* Auteur à qui adresser la correspondance : Docteur Larbi BENRAMDANE, SRMI, 228, route Ouled Ziane CASABLANCA - MAROC

(Reçu le 15 février 2001 ; accepté le 20 mars 2001)

\section{RÉSUMÉ}

Cet article décrit les méthodes de dosage et de spéciation de l'arsenic, mises au point par les auteurs dans le cadre de leurs travaux de recherche sur le dosage des espèces arséniées d'interêt toxicologique - arsenic minéral, sous forme arsénite [As(III)] et arséniate [As(V)] et ses métabolites [acide monométhylarsonique (MMA) et acide diméthylarsinique (DMA)] dans les milieux biologiques.

Trois méthodes analytiques sont décrites. Ce sont des méthodes simples, rapides, sélectives et sensibles. Elles permettent de différencier et de quantifier les espèces arséniées dans les différents milieux biologiques.

Chaque méthode est destinée à une analyse donnée :

- La première, appelée méthode Astox est destinée au dosage de l'arsenic total d'interêt toxicologique

- La deuxième, dite méthode As(III) permet de doser sélectivement As(III)

\section{SUMMARY}

This paper describes three methods of determination and speciation of arsenic, etablished by the authors during their research on the determination of arsenic species of toxicological interest - inorganic arsenic [arsenite (AsIII), arsenate $(A s V)]$ and their metabolites [ monomethylarsonic acid (MMA) and dimethylarsinic acid (DMA)] in the biological media.

These three methods are simple, fast, specific and sensitive. Each method is adapted for a definite application.

- The first method designated as "As tox method" : leads to the determination of total arsenic of toxicological interest. - The second method designated as "As III method" : permits the specific determination of AsIII.

- The third method : "As speciation method" : it permits the speciation of arsenic species by high performance liquid chromatography (HPLC). 
- La troisième, appelée méthode As spéciation permet une analyse fine par chromatographie liquide haute performance (CLHP).

- La méthode As spéciation a été appliquée à la spéciation de l'arsenic dans les tissus humains suite à une intoxication mortelle par l'anhydride arsénieux. Cette étude est à notre connaissance la première à avoir évalué la distribution des espèces arséniées dans les tissus humains.

\section{MOTS-CLÉS}

Arsenic, arsenic minéral, arsénobétaïne anhydride arsénieux, spectrométrie d'absorption atomique, chromatographie liquide haute performance, extraction, spéciation, intoxication aiguë, urine, tissus humains.

\section{Introduction}

Il est maintenant bien établi que la toxicité de l'arsenic dépend de la forme chimique du composé impliqué, que les espèces minérales [arsénite: As(III) et arséniate: $\mathrm{As}(\mathrm{V})]$ sont de loin les espèces arséniées les plus toxiques, comparées aux espèces organiques non toxiques d'origine alimentaire, notamment l'arsénobétaïne (Asbet), espèce très majoritaire dans les aliments marins (96\% de l'arsenic total dans les aliments marins) $(1,2,3)$.

Le dosage de l'arsenic total devient obsolète compte tenu des différences de toxicité des diverses formes chimiques. Les méthodes d'analyse des composés arséniés doivent être par conséquent sélectives, c'est à dire capables de doser spécifiquement l'arsenic total d'origine toxique $\mathrm{As}(\mathrm{III})$ et $\mathrm{As}(\mathrm{V})$ et leurs métabolites: acide monométhylarsonique (MMA) et acide diméthylarsinique (DMA)] voire même d'identifier et de quantifier chaque espèce dans le milieu biologique analysé. C'est pourquoi, nous avons mis au point dans le cadre de nos travaux de recherche sur l'arsenic $(3,4,5,6)$, trois méthodes analytiques complémentaires pour le dosage de l'arsenic dans les milieux biologiques. Pour les distinguer, ces méthodes ont été appelées respectivement : "méthode As tox", "méthode As(III)" et "méthode As spéciation". Chaque méthode est décrite et évaluée quant à sa maniabilité et sa sensibilité.

Nous rapportons aussi une application de la méthode As spéciation dans un cas d'intoxication mortelle par $\mathrm{As}_{2} \mathrm{O}_{3}$. Cette application est à notre connaissance la première à avoir réalisé la spéciation de l'arsenic dans les organes humains (6).

\section{Présentation et description des méthodes}

\section{Méthode As tox (5)}

\section{Principe}

La méthode As tox consiste à acidifier l'échantillon
The As speciation method was applied by the authors for arsenic speciation in the human tissues following a fatal intoxication by arsenic trioxide. This study is in our knowledge the first which evaluated the distribution of arsenic species in human organs.

\section{KEY-WORDS}

arsenic, inorganic arsenic, arsenic trioxide, arsenobétaine, atomic absorption spectrometry, high liquid performance chromatography, extraction, speciation, acute intoxication, urine human tissues.

urinaire par $\mathrm{HCl}$ concentré, et à ajouter dans ce milieu KI et l'hypophosphite de sodium. Dans ces conditions, les espèces aséniées sont réduites sous formes d'iodures d'arsines correspondantes. Ces dernières sont extraites par le toluène puis reprises en milieu aqueux par une solution à $1 \mathrm{mmol} \mathrm{H}^{-1}$ de $\mathrm{NaOH}$ avant d'être dosées par spectrométrie d'absorption atomique (SAA). Seul l'arsenic minéral et ses métabolites DMA et MMA sont extraits. Asbet, principale espèce non toxique d'origine alimentaire n'est pas extraite.

\section{Réactifs et appareillage}

\section{Réactifs}

Des solutions mères de $1 \mathrm{~g} \mathrm{l}^{-1}$ de As(III), As(V), MMA et DMA ont été préparées respectivement par dissolution dans l'eau permutée, de quantités appropriées d'anhydride arsénieux: $\mathrm{As}_{2} \mathrm{O}_{3}$ (Sigma), d'arséniate disodique: $\mathrm{Na}_{2} \mathrm{HAsO}_{4} .7 \mathrm{H}_{2} \mathrm{O}$ (Sigma), de monométhylarsonate de sodium: $\mathrm{CH}_{3} \mathrm{AsO}_{3} \mathrm{Na}_{2} .6 \mathrm{H}_{2} \mathrm{O}$ (Carlo Erba) et de diméthylarsinate de sodium (ou cacodylate de sodium): $\left(\mathrm{CH}_{3}\right)_{2} \mathrm{AsO}_{2} \mathrm{Na}_{3} \mathrm{H}_{2} \mathrm{O}$ (Sigma). Un mélange de ces quatre espèces a été aussi préparé. La solution de Asbet $\left(0,412 \mathrm{~g} \mathrm{l}^{-1} \mathrm{As}\right)$ nous a été gracieusement fournie par le CNRS Solaize (Lyon, France). Pour les étapes de réduction et d'extraction, nous avons utilisé $\mathrm{HCl} 37 \%$ (Merck), KI (Fluka) ; $\mathrm{NaPO}_{2} \mathrm{H}_{2} \cdot \mathrm{H}_{2} \mathrm{O}$ (Prolabo), et $\mathrm{NaOH}$ (Fluka).

Pour le dosage par spectrométrie d'absorption atomique en four graphite, nous avons utilisé le Triton X100 (Merck) 0,05\% pour la dilution des échantillons et la solution de $\mathrm{Ni}\left(\mathrm{NO}_{3}\right)_{2} \cdot 6 \mathrm{H}_{2} \mathrm{O}$ (Merck) $10 \mathrm{~g} \mathrm{l}^{-1}$ comme modificateur de matrice.

Une solution aqueuse étalon contenant $50 \mu \mathrm{g} \mathrm{l}^{-1} \mathrm{~d}^{\prime}$ arsenic a été préparée quotidiennement par dilution d'une solution standard d'arsenic (Spex) de $1 \mathrm{~g} \mathrm{l}^{-1}$, et des contrôles urinaires Lyphocheck niveau 1 et niveau 2 avec des concentrations d'arsenic respectivement de $50 \mu \mathrm{g} \mathrm{l}^{-1}$ et $150 \mu \mathrm{g} \mathrm{l}^{-1}$ (BioRad) ont été utilisées pour 
contrôler le spectromètre d'absorption atomique. Enfin, des urines sélectionnées pour leur faible teneur en As total $(<10 \mu \mathrm{g} / \mathrm{l})$ ont été chargées avec le mélange des espèces arséniées pour servir à l'étalonnage pendant l'étape d'extraction.

\section{Appareillage}

Le spectromètre d'absorption atomique utilisé est de type $\operatorname{Varian}^{\circledR}$ modèle SpectrAA Zeeman 220, équipé d'un four en graphite. Le système est muni d'un correcteur d'absorption non spécifique par effet Zeeman.

\section{Méthode d'extraction \\ Étude Qualitative \\ a) Les bases de la méthode}

Notre méthode d'extraction des espèces d'arsenic d'origine toxique [As(III), As(V), DMA et MMA] à partir des urines a été adaptée d'une très vieille publication de Charlot (7) : As(III) est extrait par le benzène à partir d'un milieu chlorhydrique concentré ; As(V) est extrait après addition dans le milieu chlorhydrique de KI et de l'hypophosphite de sodium. Notre adaptation de la méthode pour l'extraction des quatre espèces a été développée comme suit :

b) Étude des mécanismes impliqués dans l'extraction

Des solutions concentrées (100 $\left.\mathrm{mg} \mathrm{l}^{-1}\right)$ de As(III), $\mathrm{As}(\mathrm{V}), \mathrm{MMA}$ et DMA ont été utilisées grâce à leurs propriétés chromogènes : l'observation de la coloration des solutions a permis de suivre le processus d'extraction. Le benzène a été remplacé par le toluène, solvant moins toxique.

La nature du mécanisme impliqué dans l'extraction dépend de l'espèce :

- Arsénite : l'arsénite est extrait par le solvant organique à partir d'un milieu chlorhydrique concentré et en présence de I' conformément à la réaction suivante :

$$
\mathrm{H}_{3} \mathrm{AsO}_{3}+3 \mathrm{IH} \mathrm{AsI}_{3}+3 \mathrm{H}_{2} \mathrm{O}
$$

La réaction est complète et immédiate dans $\mathrm{HCl}$ concentré; $\mathrm{AsI}_{3}$ est jaune dans l'eau et rouge orangé dans le toluène. Si I' est absent, As(III) peut aussi être extrait, probablement par formation de $\mathrm{AsCl}_{3}$ qui est une espèce incolore.

La reprise de As(III) dans l'eau est expliquée par la réaction suivante :

$$
\left(\mathrm{AsI}_{3}\right)_{\text {toluc̀ne }}+3 \mathrm{H}_{2} \mathrm{O}\left(\mathrm{H}_{3} \mathrm{AsO}_{3}\right)_{\text {eau }}+3 \mathrm{HI}
$$

L'équilibre s'établit entre la phase organique et la phase aqueuse. Son déplacement vers la droite est fortement facilité par l'alcalinisation ; le rendement est sensiblement amélioré comparativement aux milieux acide et neutre.
- Arséniate : l'arséniate peut être extrait seulement s'il est réduit en As(III). I' est responsable de cette réduction en même temps qu'il contribue à la formation de $\mathrm{AsI}_{3}$, qui est ensuite extrait. Les réactions impliquées sont :

$$
\begin{gathered}
\mathrm{H}_{3} \mathrm{AsO}_{4}+2 \mathrm{HI} \mathrm{H}_{3} \mathrm{AsO}_{3}+\mathrm{I}_{2}+\mathrm{H}_{2} \mathrm{O}[3] \\
\mathrm{H}_{3} \mathrm{AsO}_{3}+3 \mathrm{HI} \mathrm{AsI}_{3}+3 \mathrm{H}_{2} \mathrm{O}
\end{gathered}
$$

- MMA et DMA : MMA et DMA sont extraits par le même mécanisme que celui impliquant l'arséniate parce que dans ces espèces l'arsenic est pentavalent $\mathrm{As}(\mathrm{V})$, MMA et DMA sont réduits avec formation d'arsines méthylées: $\mathbf{C H}_{\mathbf{3}} \mathbf{A s I} \mathbf{2}_{\mathbf{2}}$ et $\left(\mathbf{C H}_{\mathbf{3}}\right)_{2} \mathbf{A s I}$ (8). Ces deux espèces sont toutes les deux responsables d'une coloration brune aussi bien en milieu chlorhydrique que dans le toluène. Les équilibres réactionnels correspondants sont :

MMA :

$$
\mathrm{CH}_{3} \mathrm{AsO}(\mathrm{OH})_{2}+4 \mathrm{HI} \mathrm{CH}_{3} \mathrm{AsI}_{2}+\mathrm{I}_{2}+3 \mathrm{H}_{2} \mathrm{O}
$$

DMA :

$$
\left(\mathrm{CH}_{3}\right)_{2} \mathrm{AsO}(\mathrm{OH})+3 \mathrm{HI}\left(\mathrm{CH}_{3}\right)_{2} \mathrm{AsI}+\mathrm{I}_{2}+2 \mathrm{H}_{2} \mathrm{O}
$$

La reprise des espèces en phase aqueuse à partir du toluène est réalisée conformément à l'équilibre [2]

- Le rôle de l'hypophosphite de sodium : durant l'étude qualitative de l'extraction nous avons observé qu'en absence d'hypophosphite, $\mathrm{AsI}_{3}, \mathrm{CH}_{3} \mathrm{AsI}_{2}$ et $\left(\mathrm{CH}_{3}\right)_{2}$ AsI se décomposent lentement avec apparition d'une espèce jaune dans l'acide chlorhydrique $\left(\mathrm{I}_{3}{ }^{-}\right)$et une violette dans le toluène $\left(\mathrm{I}_{2}\right)$. Cette décomposition peut être évitée si le milieu est additionné d'un réducteur puissant comme l'ion hypophosphite. Ce dernier renforce en plus le pouvoir réducteur de KI et rend la réaction immédiate.

\section{Optimisation de la méthode d'extraction.}

Les rendements obtenus sont de l'ordre de $100 \%$ si l'extraction par le toluène est effectuée en deux étapes ( $2 \mathrm{ml}$ à chaque fois) avec une agitation de 2 minutes. La réextraction des espèces arséniées à partir du toluène est complète avec une solution de $1 \mathrm{mmol}^{-1}$ de $\mathrm{NaOH}$. Les essais avec l'arsénobétaïne dans des conditions identiques ont montré que cette espèce n'est pas extractible (5).

\section{Résultats}

\section{Méthode optimisée ("méthode As tox")}

Sur un millilitre d'urine recueilli dans un tube en poly- 
propylène est ajouté $100 \mu \mathrm{l}$ de $\mathrm{KI}$ à $3,5 \mathrm{~mol} \mathrm{l}^{-1} ; 0,5 \mathrm{ml}$ de $\mathrm{NaH}_{2} \mathrm{PO}_{2}$ à $3 \mathrm{~mol} \mathrm{l}^{-1} ; 3 \mathrm{ml}$ de $\mathrm{HCl}$ à $37 \%$ et enfin $2 \mathrm{ml}$ de toluène. L'ensemble est vigoureusement agité pendant 2 minutes, puis centrifugé 2 minutes à 3000 tpm. Le surnageant organique est prélevé et conservé et une seconde extraction est réalisée. Au volume total $(4 \mathrm{ml})$ de la phase organique recueillie est ajouté $1 \mathrm{ml}$ de $\mathrm{NaOH}$ à $1 \mathrm{mmol}^{1^{-1}}$. Ce mélange est agité 5 minutes. La phase aqueuse alcaline est prélevée, diluée au demi par une solution aqueuse de Triton X100 0,05\% puis dosée par SAA. A ce stade, l'échantillon et le modificateur de matrice $\left[\mathrm{Ni}\left(\mathrm{NO}_{3}\right)_{2}\right]$ sont injectés simultanément dans le four. Le dosage des échantillons est réalisé en utilisant les courbes d'étalonnage.

\section{Validation de la méthode"As tox"}

La méthode est linéaire et reproductible : les coefficients de régression obtenus sont de $0.9988 \pm 0.008$ et $0.9973 \pm 0.0019$ respectivement pour les essais intra et inter essais et les coefficients de variation ne dépassent pas $10 \%$.

Les rendements obtenus pour toutes les espèces sont de $100 \%$ et la limite de quantification est de $7 \mu \mathrm{g} \mathrm{l}^{-1}$. L'arsénobetaïne qui est l'espèce organique non toxique majoritaire dans les aliments marins n'est pas extractible par notre méthode (tableau I).

Tableau I : Extraction de As(III), As(V), DMA et MMA en présence de l'arsénobétaïne; dosage par SAA (5)

\begin{tabular}{|c|c|c|c|}
\hline \multicolumn{3}{|c|}{ Concentration d'arsenic dans les urines $\left(\boldsymbol{\mu g} \mathbf{~ I}^{-1}\right)$} & \\
\cline { 1 - 2 } $\begin{array}{c}\text { As(III) + (AsV) + } \\
\text { DMA+MMA }\end{array}$ & Asbet & Total & $\begin{array}{c}\text { As extrait } \\
\left(\boldsymbol{\mu g ~ ~ ^ { - 1 }}\right)\end{array}$ \\
\hline 15 & 21 & 36 & 14,4 \\
\hline 25 & 42 & 67 & 25,6 \\
\hline 50 & 42 & 92 & 50,3 \\
\hline 120 & 210 & 330 & 119 \\
\hline 125 & 105 & 230 & 124,5 \\
\hline 200 & 42 & 242 & 200,4 \\
\hline
\end{tabular}

\section{Méthode As (III)}

\section{Principe}

Cette méthode consiste à séparer As(III) du groupe As(V)-DMA-MMA par échange d'ions sur une cartouche de silice greffée avec des chaines alkyles C18 comportant des groupements ammoniums quaternaires.

\section{Matériel et réactifs}

Les cartouches utilisées sont de type Bond Elut®
(Varian) de $2,8 \mathrm{ml}$ de volume, remplies de silice greffée par des groupements alkyles C18 comportant des échangeurs d'anions de type ammonium quaternaire (SAX). Les réactifs utilisés sont : l'eau permutée, méthanol (Carlo Erba), $\mathrm{HCl}$ (Merck). Les solutions étalons des espèces arséniées étudiées ont été préparées à partir des solutions mères précédemment citées (voir paragraphe 1-2-1). L'arsenic a été dosé dans les fractions d'élution par SAA (voir paragraphe 1-2-2) en utilisant la méthode des ajouts dosés.

\section{Méthode ["méthode As(III)"]}

Un volume de $100 \mu \mathrm{l}$ à $200 \mu \mathrm{l}$ de solution aqueuse de $\mathrm{pH}$ neutre, contenant $1 \mathrm{~g} \mathrm{l}^{-1} \mathrm{~d}^{\prime}$ arsenic est placé dans une cartouche $\mathrm{C} 18$ Bond Elut de $2,8 \mathrm{ml}$ contenant un échangeur anionique de type SAX. La cartouche a été préalablement conditionnée par $3 \mathrm{ml}$ de méthanol, 1,5 $\mathrm{ml}$ de $\mathrm{HCl} 0,5 \mathrm{~N}$ et rincée par $6 \mathrm{ml}$ d'eau. L'étude de la courbe d'élution de chaque espèce arséniée [As(III), $\mathrm{As}(\mathrm{V}), \mathrm{DMA}, \mathrm{MMA}]$, a montré que As(III) est élué totalement de la cartouche par $3 \mathrm{ml}$ d'eau distillée. Les autres espèces sont éluées indifféremment par $1 \mathrm{ml}$ de $\mathrm{HCl} 0,5 \mathrm{~N}$. Pour construire les courbes d'élution, des fractions de $0,5 \mathrm{ml}$ ont été recueillies à la sortie de la cartouche et dosées par SAA. La figure 1 représente la courbe d'élution d'un mélange des quatre espèces arséniées. L'intérêt de cette méthode est le dosage spécifique de As(III) en urgence. Cette espèce étant la plus toxique et la plus impliquée dans les intoxications par l'arsenic minéral.

\section{Méthode As spéciation}

Nous avons appliqué une méthode de séparation des quatre espèces arséniées [As(III), As(V), DMA, MMA] par CLHP en mode paires d'ions et une détection par spectrométrie d'absorption atomique après recueil des fractions (6).

\section{Matériel et réactifs}

Appareillage: Le système chromatographique utilisé comprend une pompe isocratique (Shimadzu, modèle LC-6A) et une vanne d'injection Rhéodyne 7010 (Touzart et Matignon) munie d'une boucle d'échantillonnage de $20 \mu$ l. La séparation est réalisée en mode paires d'ions sur une colonne résistante aux $\mathrm{pH}$ extrêmes: SMT OD-5-100 (250 mm x 4,6 mm).

Pour les étapes de mise au point des séparations, nous avons utilisé un spectrophotomètre UV (SPD-6A, Shimadzu) muni d'une cellule de $8 \mu \mathrm{l}$. Le dosage des espèces a été effectué à l'aide d'un spectromètre d'absorption atomique après recueil des fractions (voir paragraphe 1-2-2).

Réactifs : Des solutions étalons de $1 \mathrm{~g} \mathrm{l}^{-1}$ de $\mathrm{As}(\mathrm{III})$, $\mathrm{As}(\mathrm{V}), \mathrm{MMA}$, et DMA ont été préparées en dissolvant 
les poudres correspondantes (paragraphe 1-2-1). La phase mobile a été préparée en utilisant l'eau permutée, l'hydroxyde de tétrabutylammonium (HTBA) à $40 \%$ dans l'eau (Fluka), l'acide orthophosphorique Normapur (Prolabo). Celui-ci sert à ajuster le pH à 8 après addition de HTBA.

Après chaque préparation, la phase mobile est filtrée sur filtre d'acétate de cellulose (Millipore) de 0,2 $\mu \mathrm{m}$ et dégazée par ultrasons.

\section{Méthode optimisée}

Les conditions chromatographiques optimisées conduisent à une bonne séparation des quatre espèces arséniées en moins de 17 minutes (figure 2). La bonne séparation entre les espèces arséniées a permis d'isoler chacune d'entre elles et de les doser par absorption atomique. La limite de quantification de la méthode (solution pure) est de $7 \mu \mathrm{g} \mathrm{l}^{-1}$.

L'efficacité de la méthode a été étudiée sur une solution étalon contenant les quatre espèces arséniées à une concentration de $750 \mu \mathrm{g} \mathrm{l}^{-1}$ chacune. Les taux de récupération des fractions après séparation chromatographique sont de $96,4 \% ; 94,7 ; 96,0$ et 93,7 respectivement pour As(III), DMA, MMA, et As(V).

\section{Application de la méthode de spéciation à un cas d'in- toxication mortelle par l'anhydride arsénieux}

\section{Introduction}

L'application que nous avons réalisée, a eu comme objectif l'étude de la distribution des espèces arséniées dans la plupart des organes humains suite à une intoxication mortelle par l'anhydride arsénieux. La victime est un homme de 28 ans qui est décédé trois jours après un suicide par absorption d'une dose massive de $\mathrm{As}_{2} \mathrm{O}_{3}$ (8 g environ) par voie orale (6).

Durant l'autopsie, des échantillons de la plupart des organes ont été prélevés et conservés à $-18^{\circ} \mathrm{C}$ avant analyse. Après le dosage de l'arsenic total dans chaque échantillon, nous avons réalisé la spéciation de l'arsenic dans les organes et le sang en utilisant la méthode CLHP en mode paires d'ions que nous avons optimisée (méthode As spéciation). L'analyse des espèces arséniées contenues dans les organes par CLHP, a nécessité leur mise en solution préalable. La méthode que nous avons employée à cet effet est une extraction des espèces par un mélange méthanol/eau (1:1). Le dosage des espèces arséniées est effectué par SAA dans les fractions correspondantes recueillies à la sortie de la colonne.

\section{Procédures}

\section{Préparation des échantillons}

Des échantillons d'organes (cerveau, cervelet, cœur, poumons, foie, reins, pancréas, rate, muscle, peau) ont été prélevés, coupés en petits morceaux et réduits sous forme de purée dans un mixeur (appareil de Potter). Une partie de la purée obtenue a été divisée en deu: échantillons, un pour le dosage de l'arsenic total et le second pour la spéciation. Ces échantillons d'organes ont été exactement pesés puis laissés 6 heures dans une étuve à $60^{\circ} \mathrm{C}$. Le séchage complet est obtenu après $3 \mathrm{~h}$ à $105^{\circ} \mathrm{C}$. A la fin de l'opération, les échantillons secs sont pesés plusieurs fois et remis en étuve jusqu'à un poids constant.

Dans le sang hémolysé collecté, le dosage de l'arsenic total a été réalisé directement. La spéciation a été effectuée dans le surnageant après centrifugation et filtration sur membrane millipore de $0,2 \mu \mathrm{m}$.

\section{Dosage de l'arsenic total dans les organes}

Les concentrations d'arsenic total dans les différents organes ont été mesurées par SAA dans les poudres sèches obtenues, après minéralisation par l'acide nitrique concentré à chaud.

Le contrôle de qualité de cette étape a été effectué en utilisant comme matériel de référence une poudre de muscle de roussette: DORM-2 (arsenic total: $18 \pm 1,1$ ) commercialisée par le Conseil National de Recherche du Canada.

\section{Extraction des espèces arséniées des organes}

Les espèces arséniées ont été extraites à partir des poudres d'organes obtenues en utilisant une méthode qui a été, à l'origine, employée pour l'extraction de l'arsenic à partir des tissus d'animaux marins $(9,10)$. Cette méthode d'extraction a été validée par El Moll et coll (10) en utilisant les échantillons de tissus de huit espèces différentes de poissons et fruits de mer. Son application dans le cas des organes humains nous a permis d'obtenir des rendements d'extraction compris entre 71 et $98 \%$

\section{Méthode :}

Sur une prise d'essai de 400 à $500 \mathrm{mg}$ de poudre sèche placée dans un tube en polypropylène sont ajoutés $2 \mathrm{ml}$ d'un mélange méthanol/eau (1:1). La solution est ensuite agitée pendant $20 \mathrm{mn}$, puis centrifugée à $3300 \mathrm{tpm}$. Le surnageant est conservé et l'opération est répétée quatre autres fois en renouvelant le mélange solvant. L'extrait total obtenu a été évaporé sous vide à $40^{\circ} \mathrm{C}$ à l'aide d'un évaporateur Speed Vack. Chaque résidu a été solubilisé dans un volume approprié d'eau permutée pour obtenir des concentrations d'arsenic $>800 \mu \mathrm{g} \mathrm{l}^{-1}$. Le volume d'eau ajouté à chaque résidu d'organe a été calculé d'après la valeur de la concen- 
tration de l'arsenic total dans les minéralisats. Les solutions ont été agitées $20 \mathrm{~min}$, centrifugées et filtrées sur une membrane Millipore de 0,2 $\mu \mathrm{m}$.

\section{Séparation des espèces arséniées extraites des organes}

Les extraits d'organes ont été filtrés sur membrane Millipore de $0,2 \mu \mathrm{m}$, et injectés dans la colonne. Chaque fraction de As(III), DMA, MMA et As(V) a été collectée grâce à la connaissance des temps de rétention correspondants déterminés en solution pure. Les fractions obtenues sont concentrées si nécessaire (par évaporation sous vide à $40^{\circ} \mathrm{C}$ ) puis dosées par SAA.

\section{Résultats}

\section{Concentrations d'arsenic total dans les organes}

Les concentrations d'arsenic total dans les organes (par rapport au poids sec) sont présentées dans le tableau II. Ces résultats montrent que la distribution de l'arsenic est ubiquitaire et que le foie suivi par les reins contiennent les concentrations les plus élevées (147 et $26,6 \mu \mathrm{g} / \mathrm{g}$ ). Les teneurs dans les autres organes sont relativement égales ( 8 à $12 \mu \mathrm{g} / \mathrm{g}$ ). La peau par contre, présente la plus faible concentration $(2,9 \mu \mathrm{g} / \mathrm{g})$.

\section{Concentrations des espèces arséniées dans les organes}

Les résultats sont présentés dans le tableau III. Ils montrent que dans tous les tissus, As(III) est la principale espèce rencontrée. Les teneurs en As(III) représentent entre 74 et $86 \%$ de l'arsenic dans la majorité des organes à l'exception du cervelet, du cerveau et de la peau (respectivement $49 \%, 53 \%$ et $56 \%$ ). Les proportions des métabolites (DMA et MMA) sont relativement faibles et les concentrations de $\mathrm{As}(\mathrm{V})$ sont inférieures à la limite de quantification sauf dans le foie et dans les reins.

Tableau II : Concentrations d'arsenic total dans les organes et le sang (6).

\begin{tabular}{|c|c|}
\hline organe & poids sec $(\boldsymbol{\mu g} / \mathbf{g})$ \\
\hline foie & 147 \\
reins & 26,6 \\
muscle & 12,3 \\
couur & 11,75 \\
rate & 11,72 \\
pancréas & 11,2 \\
poumons & 11,13 \\
cervelet & 10,95 \\
cerveau & 8,33 \\
peau & 2,9 \\
sang hémolysé & 0,422 \\
\hline
\end{tabular}

\section{Discussion et Conclusion}

Ce travail décrit les trois méthodes analytiques que nous avons mises au point pour l'analyse de l'arsenic en toxicologie. Elles permettent de différencier et de quantifier les espèces arséniées d'intérêt toxicologique. Par ailleurs, nous avons appliqué la méthode As spéciation dans un cas d'intoxication mortelle par l'anhydride arsénieux.

La méthode "As tox" est basée sur l'extraction liquideliquide de la somme des espèces arséniées d'intérêt toxicologique (arsénite, arséniate et leurs métabolites DMA et MMA) à partir des urines et leur dosage par spectrométrie d'absorption atomique. L'arsénobétaine qui est l'espèce arséniée non toxique majoritaire apportée par l'alimentation marine, n'est pas extractible.

L'étude des mécanismes réactionnels de réduction des espèces arséniées et l'extraction des iodures d'arsines par le toluène, a montré que la réduction des espèces arséniées pentavalentes (arséniate, DMA, MMA) est fortement améliorée par la présence de l'ion hypophosphite; l'hypophosphite augmente très significativement le pouvoir réducteur de l'espèce HI, qui a été utilisée seule dans les méthodes similaires proposées dans la littérature $(11,12)$. L'addition de l'hypophosphite augmente aussi les rendements de la réaction de réduction et de l'extraction et rend immédiate la réduction des espèces arséniées pentavalentes grâce à son pouvoir réducteur. Le pouvoir réducteur de l'hypophosphite renforce celui de $\mathrm{HI}$ et contribue à préserver les iodures d'arsines de la destruction par l'oxygène. L'autre amélioration que nous avons apportée est l'utilisation d'une solution aqueuse de $\mathrm{NaOH}$ pour la réextraction des espèces arséniées à partir du toluène. En effet, l'utilisation d'une solution alcaline augmente le rendement en déplaçant l'équilibre d'extraction vers la phase aqueuse. Par ailleurs, les propriétés chromogènes des iodures d'arsine permettent d'avoir une idée rapide sur la nature chimique de l'espèce arséniée étudiée.

Cette méthode comme celles proposées par Bavazzano et coll (11) et Lebouil et coll (12) est très simple, spécifique et très rapide. La méthode Astox permet la mise en évidence rapide d'une éventuelle intoxication par l'arsenic minéral sans avoir directement recours aux méthodes de spéciation d'arsenic, longues et coûteuses basées sur la séparation chromatographique des espèces.

La méthode "As(III)" est basée sur la séparation de As(III) parmi les quatre espèces arséniées [As(III), As(V), DMA et MMA] sur cartouche Bond Elut et le dosage des deux fractions par SAA. Dans la deuxième fraction les espèces ne sont pas séparées entre elles à cause de leurs pKa très proches. Cette méthode est simple et rapide, mais elle ne permet pas une séparation complète entre les quatre espèces. Néanmoins, elle a l'avantage d'isoler As(III) et de le doser dans un mélan- 
ge de composés arséniés. As(III) a été séparée des autres espèces grâce à son $\mathrm{pKa}$ élevé $(9,2)$ qui la rend non ionisable au $\mathrm{pH}$ utilisé ( $\mathrm{pH}$ neutre) et donc non retenue par l'échangeur anionique. Les autres espèces sont retenues sur la phase stationnaire grâce à leurs faibles pKa. Par contre, la faible différence entre ces pKa ne permet pas de les séparer dans les conditions de la méthode, et l'étude de leur séparation en jouant sur la concentration de $\mathrm{HCl}$ n'a pas apporté de résultat.

La méthode "As(III)" peut être utilisée pour évaluer une intoxication par As(III) chez l'homme dans les différents milieux biologiques. Elle permet de doser la quantité de As(III) non métabolisée et celle transformée en métabolites.

La méthode "As spéciation" est une méthode de séparation de quatre espèces arséniées [As(III), $\mathrm{As}(\mathrm{V})$, DMA et MMA] par CLHP en mode paires d'ions. Elle $s$ 'interesse donc à la séparation des espèces arséniées présentes dans l'organisme suite à une intoxication par l'arsenic minéral.
La méthode de séparation optimisée a été appliquée à la spéciation de l'arsenic dans les organes et le sang à propos d'un cas de suicide par une forte dose d'anhydride arsénieux. La spéciation de l'arsenic dans les organes a été possible grâce à l'extraction des espèces arséniées et leur mise en solution aqueuse.

Notre application est, à notre connaissance, la première à avoir effectué la spéciation de l'arsenic sur la presque totalité des organes humains dans' un cas d'intoxication aiguë fatale. Dans les intoxications expérimentales citeés dans la littérature, le foie était l'organe exclusivement étudié ; notre travail a étendu l'étude à une dizaine d'organes $(13,14)$.

L'examen des résultats a permis de noter les observations suivantes :

Arsenic total : les concentrations de l'arsenic total dans les organes confirment les résultats obtenus précédemment chez l'Homme $(15,16,17)$ : la distribution de l'arsenic dans l'organisme est ubiquitaire avec une pré-

Tableau III : Concentrations des espèces arséniées dans les organes et le sang ( $\mu g / g$, poids sec) (6).

\begin{tabular}{|c|c|c|c|c|c|c|c|}
\hline Organe & $\begin{array}{l}\operatorname{As}(\mathrm{III}) \\
\text { (a), (b) }\end{array}$ & $\begin{array}{l}\text { DMA } \\
\text { (a), (b) }\end{array}$ & $\begin{array}{c}\text { MMA } \\
\text { (a), (b) }\end{array}$ & $\begin{array}{l}\operatorname{As}(\mathbf{V}) \\
\text { (a), (b) }\end{array}$ & $\mathbf{M b}^{*}+\mathbf{A s}(\mathbf{V})$ & $\begin{array}{c}\mathbf{A s}(\mathrm{III}) / \mathbf{M b}^{*} \\
+\mathbf{A s}(\mathbf{V})\end{array}$ & MMA/DMA \\
\hline foie & $\begin{array}{l}122,00 \\
(84 \%)\end{array}$ & $\begin{array}{l}5,91 \\
(4 \%)\end{array}$ & $\begin{array}{c}14,7 \\
(10 \%)\end{array}$ & $\begin{array}{l}2,94 \\
(2 \%)\end{array}$ & $\begin{array}{l}23,55 \\
(16 \%)\end{array}$ & 5 & 2,5 \\
\hline reins & $\begin{array}{l}19,25 \\
(74 \%)\end{array}$ & $\begin{array}{c}1,80 \\
(7 \%)\end{array}$ & $\begin{array}{c}4,32 \\
(17 \%)\end{array}$ & $\begin{array}{c}0,53 \\
(2 \%)\end{array}$ & $\begin{array}{c}6,65 \\
(26 \%)\end{array}$ & 3 & 2,4 \\
\hline rate & $\begin{array}{c}9,50 \\
(81 \%)\end{array}$ & $\begin{array}{l}0,59 \\
(5 \%)\end{array}$ & $\begin{array}{c}1,65 \\
(14 \%)\end{array}$ & $<\mathrm{LD}$ & $\begin{array}{c}2,24 \\
(19 \%)\end{array}$ & 4 & 2,8 \\
\hline poumons & $\begin{array}{c}9,46 \\
(86 \%)\end{array}$ & $\begin{array}{l}0,45 \\
(4 \%)\end{array}$ & $\begin{array}{c}1,11 \\
(10 \%)\end{array}$ & $<\mathrm{LD}$ & $\begin{array}{c}1,56 \\
(15 \%) \\
\end{array}$ & 6 & 2,5 \\
\hline pancréas & $\begin{array}{c}9,18 \\
(82 \%)\end{array}$ & $\begin{array}{c}0,45 \\
(5 \%)\end{array}$ & $\begin{array}{c}1,34 \\
(10 \%)\end{array}$ & $<\mathrm{LD}$ & $\begin{array}{c}1,79 \\
(14 \%)\end{array}$ & 5 & 3 \\
\hline muscle & $\begin{array}{c}9,17 \\
(77 \%)\end{array}$ & $\begin{array}{c}0,73 \\
(6 \%)\end{array}$ & $\begin{array}{c}1,96 \\
(17 \%)\end{array}$ & $<\mathrm{LD}$ & $\begin{array}{c}2,69 \\
(23 \%)\end{array}$ & 3 & 2,7 \\
\hline cœur & $\begin{array}{c}9,05 \\
(77 \%)\end{array}$ & $\begin{array}{c}0,64 \\
(5 \%)\end{array}$ & $\begin{array}{c}1,61 \\
(14 \%)\end{array}$ & $<\mathrm{LD}$ & $\begin{array}{c}2,25 \\
(19 \%)\end{array}$ & 4 & 2,5 \\
\hline cervelet & $\begin{array}{c}5,15 \\
(49 \%)\end{array}$ & $\begin{array}{c}1,60 \\
(15 \%)\end{array}$ & $\begin{array}{c}3,76 \\
(36 \%)\end{array}$ & $<\mathrm{LD}$ & $\begin{array}{c}5,36 \\
(51 \%)\end{array}$ & 1 & 2,4 \\
\hline cerveau & $\begin{array}{c}4,21 \\
(53 \%)\end{array}$ & $\begin{array}{c}1,10 \\
(14 \%)\end{array}$ & $\begin{array}{c}2,65 \\
(33 \%)\end{array}$ & $<\mathrm{LD}$ & $\begin{array}{c}3,75 \\
(47 \%)\end{array}$ & 1 & 2,4 \\
\hline peau & $\begin{array}{c}1,62 \\
(56 \%)\end{array}$ & $\begin{array}{c}0,34 \\
(12 \%)\end{array}$ & $\begin{array}{c}0,91 \\
(32 \%)\end{array}$ & $<\mathrm{LD}$ & $\begin{array}{c}1,25 \\
(44 \%)\end{array}$ & 1 & 2,7 \\
\hline $\begin{array}{c}\text { sang } \\
\text { hémolysé } \\
(\mu \mathrm{g} / \mathrm{g})\end{array}$ & $\begin{array}{c}0,224 \\
(53 \%)\end{array}$ & $\begin{array}{c}0,0539 \\
(13 \%)\end{array}$ & $\begin{array}{c}0,135 \\
(32 \%)\end{array}$ & $\begin{array}{l}0,009 \\
(2 \%)\end{array}$ & $\begin{array}{c}0,198 \\
(47 \%)\end{array}$ & 1 & 2,5 \\
\hline
\end{tabular}

$\left(^{*}\right)$ : pourcentage par rapport à la somme des concentrations $\mathrm{Mb}^{*}=\mathrm{DMA}+\mathrm{MMA}$ (a) : valeurs moyennes $(\mathrm{n}=3)$

(b) : valeurs corrigées (Rendement $=100 \%$ ) 
dominance dans le foie suivi des reins. Cette prédilection pour le foie et les reins peut être expliquée par le rôle de ces organes respectivement dans la détoxication et l'élimination de l'arsenic $(3,17,18)$. Par ailleurs, les niveaux des concentrations d'arsenic dans les différents organes concordent avec les symptomes observés lors des intoxications aiguës (insuffisances hépatique et rénale, effets cardiovasculaires, rhabdomyolyse et atteinte neurologique) $(6,16,17)$.

Spéciation de l'arsenic : les résultats que nous avons obtenus confirment ceux trouvés par expérimentation chez l'animal $(13,14)$. Ils démontrent que As(III) est de loin l'espèce arséniée majoritaire liée aux tissus. Les concentrations des métabolites mesurées dans la majorité des tissus sont faibles par rapport à As(III). Ceci s'explique par l'élimination rapide des métabolites par le sang après leur formation hépatique.

Les rapports de As(III) aux autres espèces calculés pour les différents organes ont montré des résultats faibles concernant le cerveau et le cervelet. Ceci est probable-

\section{Références}

1. Arbouine M.W., Wilson H.K. The effect of seafood consumption on the assessment of occupational exposure to arsenic by urinary arsenic speciation measurements. J. Tr. Element Electrolytes Hlth. Diseaes, 1992 ; 6 : 153-160.

2. Farmer J.H., Johnson L.R. Assessment of occupational exposure to inorganic arsenic based on urinary concentrations and speciation of arsenic. Br. Med. J. $1990 ; 47$ : 342-348.

3. Benramdane L., Bressolle F., Vallon J.J. Arsenic speciation in humans and food products: A review. J. Chromatogr. Sc. 1999 ; 37 : 330-344.

4. Benramdane L., Accominotti M., Vallon J.J. Dosage de l'arsenic minéral dans les milieux biologiques par CLHP avec détection ampérométrique après réduction de l'arséniate en arsénite et extraction par le toluène. Toxicorama, 1997 ; numéro spécial : 346-351.

5. Benramdane L., Accominotti M., Vallon J.J. Validated determination of total arsenic species of toxicological interest ( arsenite, arsenate, and metabolites) by atomic absorption spectrometry after separation from dietary arsenic by liquid extraction: toxicological applications. Analyst, $1998 ; 123$ : 1711-1715.

6. Benramdane L., Accominotti M., Fanton L., Malicier D., Vallon J.J. Arsenic speciation in human organs following fatal arsenic trioxide poisoning - case report. Clin. Chem. 1999 ; 45 : 301-306.

7. Les réactions chimiques en solution aqueuse et caractérisation des ions/ in. Charlot G., ed Paris: Masson $7^{\mathrm{cmc}}$ édn., 1983, pp. 272-276.

8. Nouveau traité de chimie minérale/ in. Pascal P. ed. Paris: Masson, 1958, vol. 9, pp. 474-494.

9. Alberti J., Rubio R., Rauret G. Extraction method for arsenic speciation in marine organisms. Fres. J. Anal. Chem., $1995 ; 351: 420-425$. ment dû à l'affinité des métabolites méthylés pour les tissus riches en lipides, par rapport à As(III).

Comparativement aux tissus, le sang est bien moins concentré en arsenic total (il y a une clearance rapide de l'élimination de l'arsenic du sang). Ceci doit être pris en compte pour évaluer un résultat de dosage de l'arsenic dans le sang.

L'étude du rapport MMA/DMA pour les organes et le sang a montré que le MMA était en moyenne 3 fois plus concentré que le DMA. Ce résultat est une caractéristique des intoxications aiguës $(17,18)$.

La présence de $\operatorname{As}(\mathrm{V})$ complète le schéma de transformation de $A s(I I I)$ in vivo, défini d'après les résultats apportés de la littérature $(6,13-18)$ : $\mathbf{A s}(\mathbf{V})$ As(III) $8<$ MMA $\&$ DMA. La transformation de As(III) en métabolites monométhyle (MMA) et diméthylé (DMA) est relativement bien élucidée. Par contre, le mécanisme d'oxydation de As(III) en As(V) reste à définir.

10. El Moll A., Heimburger R., Lagarde F., Leroy M.J.F., Maier E. Arsenic speciation in marine organisms: from the analytical methodology to the constitution of reference materials. Fres. J. Anal. Chem., 1996 ; 354 : 550-556.

11: Bavazzano P., Perico A., Rosendahl K., Apostoli P. Determination of urinary arsenic by solvent extraction and electrothermal atomic absorption spectrometry. A comparison with directly coupled high performance liquide chromatography-inductively coupled plasma mass spectrometry.J. Anal. Atom. Spectrom., $1996 ; 11$ : 521-524.

12. Lebouil A., Notelet S., Cailleux A., Turcant A., Allain P. Dosage de $I^{\prime}$ arsenic urinaire après séparation de $l^{\prime}$ arsenobétaïne. Toxicorama, $1997 ; 9: 171-176$.

13. Bogdan G.M., Sampayo-Rayes A., Aposhian H.V. Arsenic binding proteins of mammalian systems: Isolation of three arsenite binding proteins of rabbit liver. Toxicology, 1994 ; 93 : 175-193.

14. Marafante E ., Rade J., Sabbioni E., Bertolero F., Foa V. Intracellular interaction and metabolic fate of arsenite in the rabbit. Clin. Toxicol., $1981 ; 18: 1335-1341$.

15. Ghariani M., Adrien M.L., Raucoules M., Bayle J., Jacomet Y., Grimaud D. Intoxication suraiguë à l'arsenic. Ann. Fr. Anesth. Réanim., 1991 ; 10 : 304-307.

16. Wattel F., Chopin C., Haguenoer J.M., Gosselin B., Durocher A., Bouchez B. Intoxication par ingestion d'arsenic et ses dérivés. A propos de trois observations. Lille Médical, 1979 ; 24 : 703-706.

17. Mathieu P., Buchet J.P., Roels H.A., Lauwerys R., The metabolism of arsenic in humans acutely intoxicated by As2O3. Its significance for the duration of BAL therapy. Clin. Toxicol., $1981 ; 18: 1067-1075$.

18. Lovell M.A., Farmer J.G. Arsenic speciation in urine from human intoxicated by inorganic arsenic compounds. Human Toxicol., 1985 ; 4 : 203-214. 